\title{
Evaluación de Modelos Térmicos usados para Estimar la Temperatura del Punto Caliente en Transformadores de Distribución
}

\author{
Daniel F. Martínez, Alexander Céspedes* y Diego F. García \\ Escuela de Ingeniería Eléctrica y Electrónica, Facultad de Ingeniería, Universidad del Valle, Calle 13 \#100-00, \\ Cali - Colombia (e-mail: daniel.martinez@correounivalle.edu.co; alexander.cespedes@correounivalle.edu.co; \\ diego.garcia@correounivalle.edu.co).
}

* Autor a quien debe ser dirigida la correspondencia

Recibido Jun. 11, 2018; Aceptado Ago. 13, 2018; Versión final Ene. 3, 2019, Publicado Jun. 2019

\section{Resumen}

Este artículo presenta una evaluación de los principales modelos térmicos de transformadores considerando el número de datos de entrada requeridos y la confiabilidad de los datos de salida de cada modelo. Además, se presenta un breve análisis del comportamiento térmico del transformador basado en la temperatura del punto caliente. Luego, se describe una validación experimental de los modelos térmicos realizada en dos transformadores de distribución inmersos en aceite vegetal, simulando un perfil de carga en periodos de tiempo específicos. Finalmente, los resultados experimentales son comparados con datos resultantes de la implementación de los modelos en un software de análisis numérico con el fin de establecer las diferencias paramétricas y la precisión de cada modelo, permitiendo concluir que el desempeño más óptimo corresponde al modelo de ecuaciones diferenciales de la Comisión Electrotécnica Internacional..

Palabras clave: modelos térmicos; perfil de carga; temperatura de punto caliente; transformadores inmersos en aceite; evaluación.

\section{Evaluation of Thermal Models used to Estimate the Hot-Spot Temperature in Distribution Transformers}

\begin{abstract}
This paper presents an evaluation of the main transformers thermal models considering the number of input data required and the output data reliability of each model. Also, a brief analysis of transformer thermal behaviour based on the hot-spot temperature is shown. Then, an experimental validation of the thermal models performed in two oil-immersed distribution transformers is described, simulating a load profile in specific periods of time. Finally, the experimental results are compared with data resulting from the implementation of the models in numerical analysis software in order to establish the parametric differences and precision of each model showing that the most optimal performance corresponds to the model of differential equations of the International Electrotechnical Commission.
\end{abstract}

Keywords: thermal models; load profile; hot-spot temperature; oil-immersed transformers; assessment. 


\section{INTRODUCCIÓN}

El transformador es un equipo indispensable en los sistemas de generación, transmisión y distribución de energía eléctrica. Un fallo o irregularidad en dicho dispositivo puede comprometer el funcionamiento de todo el sistema, lo que generaría altos costos no solo por la reparación y reemplazo de los equipos afectados, sino por la demanda que deja de ser atendida y las penalizaciones por la interrupción del servicio (Susa, 2006). Debido a lo anterior, es importante conocer y predecir el estado operativo del transformador, teniendo en cuenta que en él existen muchos factores que causan deterioro en su aislamiento y que influyen en el efecto acumulativo de la temperatura en un determinado tiempo (Santisteban et al., 2017; Biçen et al., 2011). La temperatura de punto caliente es uno de los parámetros más críticos cuando se definen las condiciones térmicas de un transformador (Tang y Wu, 2011; Daghrah et al., 2016), debido a que es uno de los factores que afectan directamente la degradación de sus aislamientos y por ende la vida útil del mismo (Cerón et al., 2015). En consecuencia, la temperatura es una variable que debe permanecer controlada durante la operación de un transformador (Del Vecchio et al., 2010). Con el crecimiento de la demanda eléctrica, y con el fin de satisfacer la misma, se incrementa la carga de los transformadores por encima de su capacidad nominal, lo cual implica que se rebase el límite de temperatura de operación, lo cual que puede afectar al material dieléctrico del transformador (Takami et al., 2007; Taheri et al., 2008).

El presente artículo tiene como objetivo evaluar los modelos térmicos de ecuaciones exponenciales y ecuaciones diferenciales (UNE-IEC, 2010; IEEE, 2011) utilizados para el análisis termodinámico de transformadores de potencia. Lo anterior con el fin de conocer cuál de ellos resulta ser el más preciso al estimar la temperatura de punto caliente de un transformador de distribución, teniendo en cuenta la cantidad de datos de entrada que exige cada uno de los diferentes modelos. La validación respectiva fue efectuada a través de la comparación de los resultados obtenidos durante la ejecución de ensayos de elevación de temperatura en dos transformadores sumergidos en aceite vegetal, y los resultados obtenidos mediante las ecuaciones que rigen cada modelo. A continuación, se describen tres modelos que estiman la temperatura de punto caliente al interior de un transformador, luego se presentan las pruebas experimentales realizadas en dos transformadores de distribución sumergidos en aceite vegetal y finalmente, se efectúa la comparación y análisis entre los resultados obtenidos con la medición real y los modelos descritos.

\section{MODELOS PARA EL CÁLCULO DE LA TEMPERATURA DE PUNTO CALIENTE}

En esta sección, se presenta una breve explicación de los modelos de ecuaciones exponenciales y ecuaciones diferenciales, de acuerdo con la Comisión Electrotécnica Internacional y el Instituto de Ingenieros Eléctricos y Electrónicos (IEC e IEEE respectivamente, por sus siglas en ingles).

\section{Modelo de ecuaciones Exponenciales de IEC}

En este modelo (UNE-IEC, 2010), la temperatura de punto caliente se determina como sigue:

$$
\theta_{\mathrm{h}}=\theta_{\mathrm{ai}}+\Delta \theta_{\mathrm{o}}+\Delta \theta_{\mathrm{h}}
$$

Donde $\theta_{\text {ai }}$ es la temperatura ambiente promedio del intervalo estudiado, $\Delta \theta_{\circ}$ es la elevación de la temperatura del aceite superior y $\Delta \theta_{\mathrm{h}}$ es la elevación de la temperatura de punto caliente respecto a la del aceite superior. El cálculo de $\Delta \theta_{\circ}$ y $\Delta \theta_{\mathrm{h}}$ dependerá de la etapa de carga que se está estudiando, ya sea para la etapa inicial de carga o cuando la etapa estudiada tiene un aumento de carga con relación a la etapa anterior, utilizando (2) y (3).

$$
\begin{aligned}
& \Delta \theta_{\mathrm{o}}=\Delta \theta_{\text {oi }}+\left\{\Delta \theta_{\text {or }} \times\left[\frac{1+\mathrm{R} \times \mathrm{K}^{2}}{1+\mathrm{R}}\right]^{\mathrm{x}}-\Delta \theta_{\text {oi }}\right\} \times\left(1-\mathrm{e}^{\frac{-\mathrm{t}}{\mathrm{k}_{11} \times \tau_{\mathrm{o}}}}\right) \\
& \Delta \theta_{\mathrm{h}}=\Delta \theta_{\mathrm{hi}}+\left\{\mathrm{H}_{\mathrm{g}_{\mathrm{r}}} \mathrm{k}^{\mathrm{y}}-\Delta \theta_{\mathrm{hi}}\right\} \times\left(\mathrm{k}_{21} \times\left(1-\mathrm{e}^{\frac{-\mathrm{t}}{\mathrm{k}_{22} \times \tau_{\mathrm{w}}}}\right)-\left(\mathrm{k}_{21}-1\right) \times\left(1-\mathrm{e}^{\frac{-\mathrm{t}}{\tau_{\mathrm{o}} / \mathrm{k}_{22}}}\right)\right)
\end{aligned}
$$

Sin embargo, cuando la etapa estudiada tiene una disminución de carga respecto a la etapa anterior se utilizan (4) y (5).

$$
\Delta \theta_{\mathrm{h}}=\mathrm{Hg}_{\mathrm{r}} \mathrm{k}^{\mathrm{y}}
$$


Donde $\Delta \theta_{\mathrm{oi}}, \Delta \theta_{\mathrm{hi}}$ son las elevaciones de temperatura del aceite superior y la temperatura de punto caliente al inicio respectivamente, $\Delta \theta_{\text {or }}$ es la elevación de la temperatura del aceite superior a pérdidas totales, $\tau_{0}$ y tw son las constantes de tiempo para la respuesta térmica del aceite y el devanado, $k$ es el factor de carga; dado por el cociente entre la potencia suministrada sobre la potencia nominal, $R$ es la relación entre el valor de pérdidas bajo carga y pérdidas sin carga, $\mathrm{k}_{11}, \mathrm{k}_{21}$ y $\mathrm{k}_{22}$ son constantes empíricas propias del modelo, $\mathrm{x}$ e y son constantes empíricas y su valor depende del tipo de refrigeración del transformador, según la Tabla 1 (IEEE, 2011).

Tabla 1: Exponentes utilizados por IEC e IEEE

\begin{tabular}{|l|c|c|c|c|}
\hline \multirow{2}{*}{ Tipo de refrigeración } & \multicolumn{2}{|c|}{ IEEE } & \multicolumn{2}{c|}{ IEC } \\
\cline { 2 - 5 } & $\mathrm{n}$ & $\mathrm{m}$ & $\mathrm{x}$ & $\mathrm{y}$ \\
\hline ONAN & 0,8 & 0,8 & 0,8 & 1,6 \\
\hline ONAF & 0,9 & 0,8 & 0,8 & 1,3 \\
\hline OFAF, OFWF & 0,9 & 0,8 & 1 & 1,3 \\
\hline ODAF, ODWF & 1 & 1 & 1 & 2 \\
\hline
\end{tabular}

\section{MODELO DE ECUACIONES EXPONENCIALES DE IEEE}

En este modelo, la temperatura de punto caliente se determina de la siguiente manera:

$$
\theta_{\mathrm{H}}=\theta_{\mathrm{A}}+\Delta \theta_{\mathrm{TO}}+\Delta \theta_{\mathrm{H}}
$$

Donde $\theta_{\mathrm{A}}$ es la temperatura ambiente, $\Delta \theta_{\text {Tо }}$ es la elevación de la temperatura del aceite superior sobre la temperatura ambiente y $\Delta \theta_{\mathrm{H}}$ la elevación de la temperatura de punto caliente sobre la del aceite superior. Estas dos variables calculan a partir de las siguientes ecuaciones:

$$
\begin{aligned}
& \Delta \theta_{\mathrm{TO}}=\left(\Delta \theta_{\mathrm{TO}, \mathrm{U}}-\Delta \theta_{\mathrm{TO}, \mathrm{i}}\right) \times\left(1-\mathrm{e}^{-\frac{\mathrm{t}}{\tau_{\mathrm{TO}}}}\right)+\Delta \theta_{\mathrm{TO}, \mathrm{i}} \\
& \left.\Delta \theta_{\mathrm{TO}, \mathrm{U}}=\Delta \theta_{\mathrm{TO}, \mathrm{R}}\left[\frac{\mathrm{K}^{2} \mathrm{R}+1}{\mathrm{R}+1}\right]^{\mathrm{n}}\right]^{\Delta \theta_{\mathrm{H}}=\left(\Delta \theta_{\mathrm{H}, \mathrm{U}}-\Delta \theta_{\mathrm{H}, \mathrm{i}}\right) \times\left(1-\mathrm{e}^{-\frac{\mathrm{t}}{\tau_{\mathrm{w}}}}\right)+\Delta \theta_{\mathrm{H}, \mathrm{i}}} \\
& \Delta \theta_{\mathrm{H}, \mathrm{U}}=\Delta \theta_{\mathrm{H}, \mathrm{R}} \mathrm{K}^{2 \mathrm{~m}} \\
& \Delta \theta_{\mathrm{H}, \mathrm{R}}=\Delta \theta_{\mathrm{H} / \mathrm{A}, \mathrm{R}}-\Delta \theta_{\mathrm{TO}, \mathrm{R}}
\end{aligned}
$$

Donde $\Delta \theta_{\mathrm{To}, \mathrm{i}}, \Delta \theta_{\mathrm{H}, \mathrm{i}}$ son las elevaciones de la temperatura del aceite superior y la temperatura de punto caliente al inicio respectivamente, $\Delta \theta$ то, $\mathrm{R}$ es la elevación de la temperatura del aceite superior durante pérdidas totales, $\tau_{0} \mathrm{y} \tau_{\mathrm{w}}$ son las constantes de tiempo del aceite y el devanado respectivamente, $\mathrm{K}$ es el factor de carga dado por el cociente entre la potencia suministrada sobre la potencia nominal, $\mathrm{R}$ es la relación entre el valor de pérdidas bajo carga y pérdidas sin carga, $\mathrm{n}$ y $\mathrm{m}$ son constantes empíricas y su valor depende del tipo de refrigeración del transformador, según la Tabla 1 (IEEE, 2011).

\section{Modelo de ecuaciones diferenciales de IEC}

En este modelo (UNE-IEC, 2010), para la estimación de la temperatura del punto caliente, se establece lo siguiente:

$$
\theta_{\mathrm{h}}=\theta_{\mathrm{o}}+\Delta \theta_{\mathrm{h}}
$$

Sin embargo, para su solución, es necesario primero calcular como condiciones iniciales. 


$$
\begin{aligned}
& \theta_{\mathrm{o}(0)}=\left[\frac{1+\mathrm{R} \times \mathrm{k}^{2}}{1+\mathrm{R}}\right] \times \Delta \theta_{\mathrm{or}}+\theta_{\mathrm{a}} \\
& \theta_{\mathrm{h} 1(0)}=\mathrm{k}_{21} \times \mathrm{K}^{\mathrm{y}} \times \Delta \theta_{\mathrm{hr}} \\
& \theta_{\mathrm{h} 2(0)}=\left(\mathrm{k}_{21}-1\right) \times \mathrm{K}^{\mathrm{y}} \times \Delta \theta_{\mathrm{hr}}
\end{aligned}
$$

A partir de estas condiciones iniciales, se resuelven las ecuaciones diferenciales:

$$
\begin{aligned}
& \mathrm{D} \theta_{\mathrm{o}}=\frac{\mathrm{D}_{\mathrm{t}}}{\mathrm{k}_{11} \tau_{\mathrm{o}}}\left[\left[\frac{1+\mathrm{R} \times \mathrm{k}^{2}}{1+\mathrm{R}}\right]^{\mathrm{x}} \times\left(\Delta \theta_{\mathrm{or}}\right)-\left[\theta_{\mathrm{o}}-\theta_{\mathrm{a}}\right]\right] \\
& \theta_{\mathrm{o}(\mathrm{t})}=\theta_{\mathrm{o}(\mathrm{t}-\mathrm{D})}+\mathrm{D} \theta_{\mathrm{o}(\mathrm{t})} \\
& \mathrm{D} \Delta \theta_{\mathrm{h} 1}=\frac{\mathrm{D}_{\mathrm{t}}}{\mathrm{k}_{22} \tau_{\mathrm{w}}} \times\left[\mathrm{k}_{21} \times \Delta \theta_{\mathrm{hr}} \mathrm{k}^{\mathrm{y}}-\Delta \theta_{\mathrm{h} 1}\right] \\
& \mathrm{D} \Delta \theta_{\mathrm{h} 2}=\frac{\mathrm{D}_{\mathrm{t}}}{\left(1 / \mathrm{k}_{22}\right) \tau_{\mathrm{o}}} \times\left[\left(\mathrm{k}_{21}-1\right) \times \Delta \theta_{\mathrm{hr}} \mathrm{k}^{\mathrm{y}}-\Delta \theta_{\mathrm{h} 2}\right] \\
& \Delta \theta_{\mathrm{h} 1}=\Delta \theta_{\mathrm{h} 1(\mathrm{t}-\mathrm{D})}+\mathrm{D} \Delta \theta_{\mathrm{h} 1(\mathrm{t})} \\
& \Delta \theta_{\mathrm{h} 2}=\Delta \theta_{\mathrm{h} 2(\mathrm{t}-\mathrm{D})}+\mathrm{D} \Delta \theta_{\mathrm{h} 2(\mathrm{t})} \\
& \Delta \theta_{\mathrm{h}(\mathrm{t})}=\Delta \theta_{\mathrm{h} 1(\mathrm{t})}+\Delta \theta_{\mathrm{h} 2(\mathrm{t})}
\end{aligned}
$$

Donde $\Delta \theta_{\text {or }}$ es la elevación de la temperatura del aceite superior durante pérdidas totales, to y tw son las constantes de tiempo del aceite y el devanado respectivamente, $\mathrm{D}$ es un operador diferencial, $\mathrm{k}$ es el factor de carga dado por el cociente entre la potencia suministrada sobre la potencia nominal, $R$ es la relación entre el valor de pérdidas bajo carga y pérdidas en vacío, $k_{11}, k_{21}$ y $k_{22}$ son constantes empíricas propias del modelo, $x$ e y son constantes empíricas y su valor depende del tipo de refrigeración del transformador, según la Tabla 1 (IEEE, 2011).

\section{ENSAYOS ELÉCTRICOS}

Para la realización de los ensayos se utilizaron dos transformadores de distribución monofásicos sumergidos en éster vegetal, con potencias de 15 kVA y 37.5 kVA y tensiones de operación de 7620/240 V. La Tabla 2 muestra los valores nominales de los transformadores mencionados según datos del fabricante.

Tabla 2: Valores nominales de los transformadores bajo prueba

\begin{tabular}{|l|r|l|r|}
\hline Potencia (VA) & 15000 & Potencia (VA) & 37500 \\
\hline Voltaje primario (V) & 7620 & Voltaje primario (V) & 7620 \\
\hline Voltaje secundario (V) & 240 & Voltaje secundario (V) & 240 \\
\hline Corriente primaria (A) & 1,969 & Corriente primaria (A) & 4,921 \\
\hline Corriente secundaria (A) & 62.5 & Corriente secundaria (A) & 156.2 \\
\hline
\end{tabular}

Estos transformadores se instrumentaron con cuatro sensores de temperatura de fibra óptica y un termopar. De acuerdo a (Navas, 2013) su instalación fue realizada desde fábrica, previa determinación de los puntos de medición, obtenidos a través de simulaciones con el método de elementos finitos en un software especializado para tal fin. Dichos sensores se encuentran ubicados uno por encima del fondo del tanque, otro por debajo del nivel de aceite, dos en los devanados (uno en el de alta tensión y otro en el de baja tensión) y uno en el núcleo, como se muestra en la Fig. 1. 
S1: Temperatura superior del aceite

S2: Temperatura inferior del aceite

S3: Temperatura devanado de alta tensión

S4: Temperatura devanado de baja tensión

T1: Temperatura en el núcleo

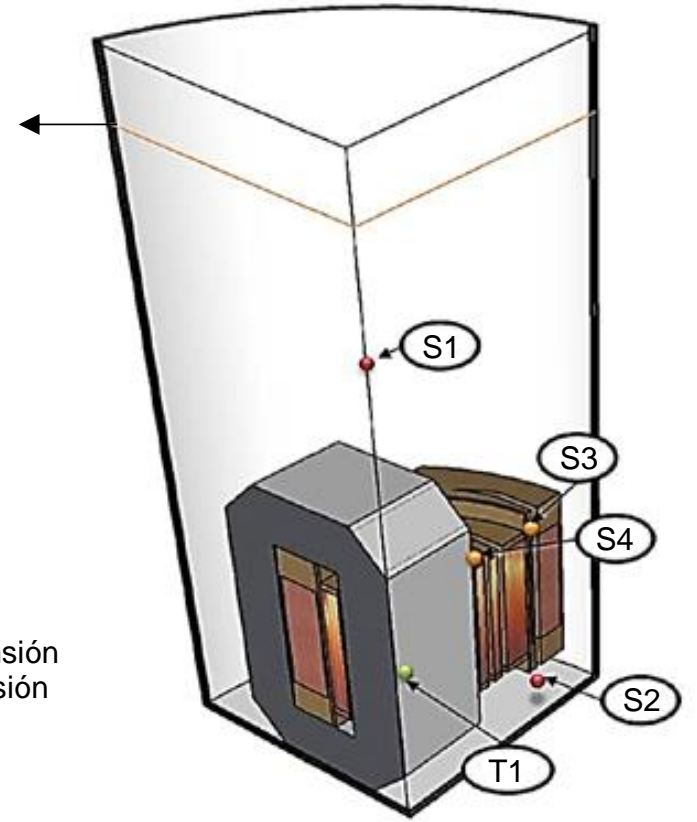

Fig.1: Ubicación de los sensores en los trasformadores bajo prueba. (Adaptado de Navas et al., 2013)

Se realizaron ensayos de rutina para determinar y verificar los parámetros necesarios a incorporar en los modelos implementados, tales como: resistencia de aislamiento (según IEEE C57.12.90), resistencia de devanados (según NTC 375), relación de transformación (según NTC 471), pérdidas sin carga y corriente de excitación (según NTC 1031) y pérdidas con carga y tensión de corto circuito (según NTC 1005). Estos ensayos tuvieron resultados satisfactorios.

Luego, se procedió a realizar ensayos de elevación de temperatura utilizando los sensores mostrados en la Fig. 1, con el objetivo de obtener la temperatura de punto caliente de los transformadores y conseguir datos que fueran comparables con los obtenidos por medio de los modelos. Los ensayos de elevación de temperatura se realizaron por medio del método de cortocircuito, simulando la carga por medio de las corrientes circulantes por los devanados. Para estos ensayos se empleó un sistema para la adquisición y registro de datos como muestra el diagrama de la Fig. 2.

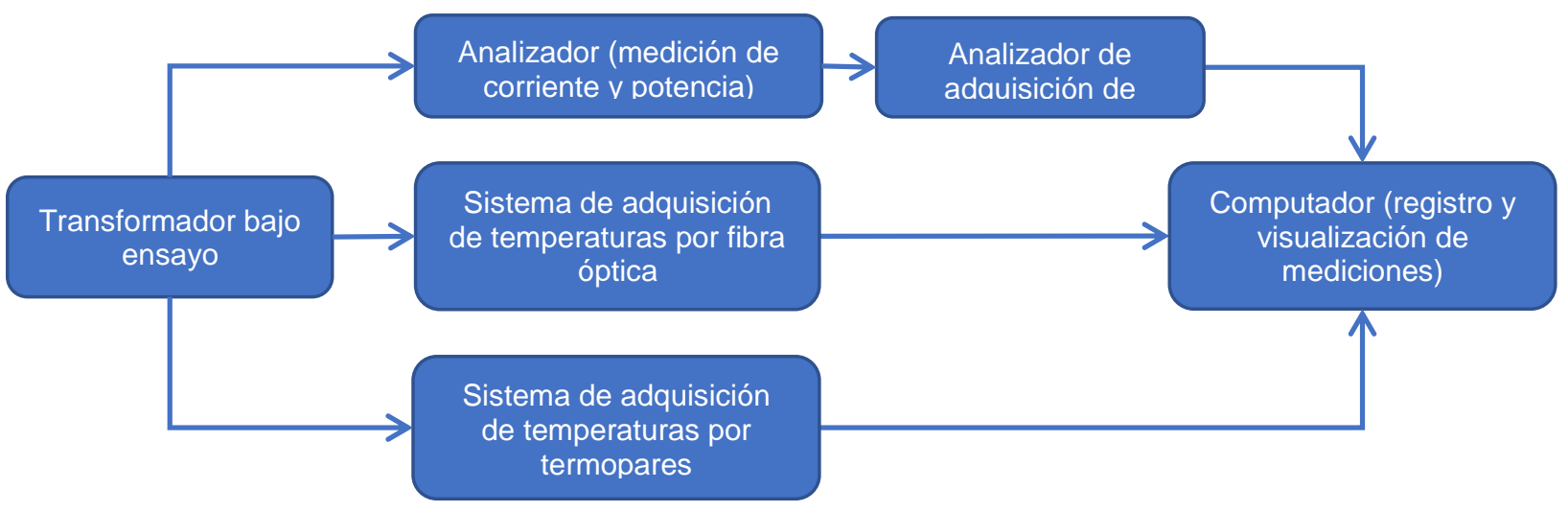

Fig.2: Adquisición y registro de variables durante el ensayo de elevación de temperatura

Al efectuar los ensayos se registraron para cada transformador nueve mediciones de temperatura en diferentes puntos, tres de ellos en el interior del transformador por medio de sensores de fibra óptica, las otras seis mediciones de temperatura fueron registradas a través del módulo de temperatura multiplexado para medición a través de termopares. En la Fig. 3 y Fig. 4 se muestran las temperaturas obtenidas en cada transformador. La temperatura ambiente reportada para cada uno de los ensayos corresponde al promedio de la temperatura de tres termopares llenos de aceite ubicados en tres puntos alrededor del transformador.

La temperatura inferior en la superficie exterior y la temperatura superior en la superficie exterior, fueron medidas por medio de termopares adheridos en la pared exterior del transformador. En cada transformador, se usaron diferentes niveles de carga, con el fin de observar la elevación y disminución de la temperatura 
ante estos diferentes cambios, sin embargo, se decidió no llevar los transformadores a condiciones de sobrecarga, para no afectar significativamente el estado actual de sus aislamientos. De las temperaturas registradas, la que presentó mayor elevación en cada ensayo fue la que corresponde al sensor de fibra óptica que está ubicado en el devanado de menor tensión, por lo tanto, se tomó esta temperatura como la temperatura de punto caliente.

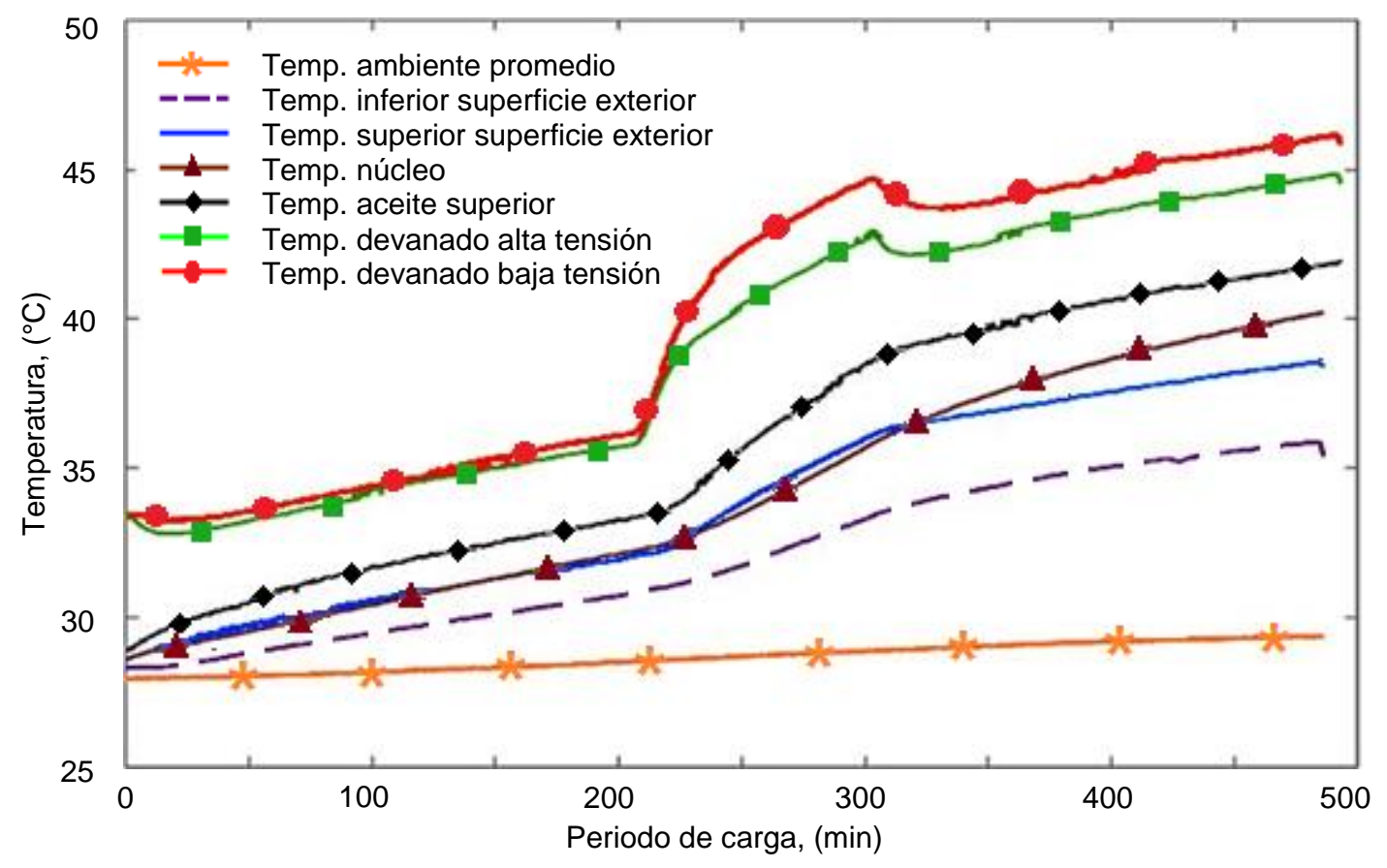

0,9

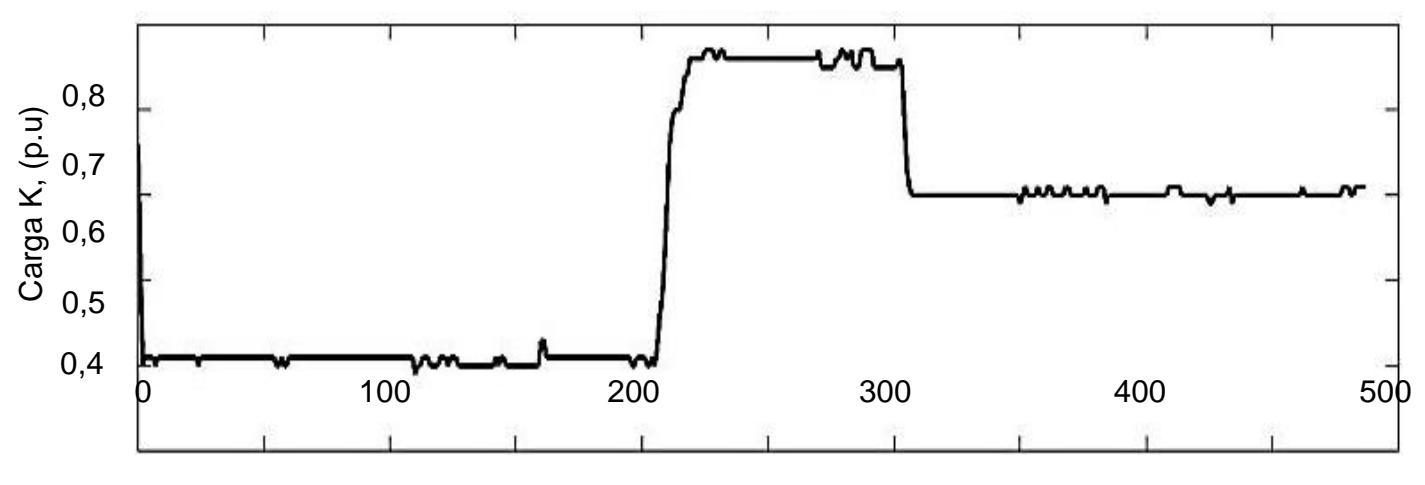

Periodo de carga, (min)

Fig.3: Transformador $15 \mathrm{kVA}$, registro de temperaturas

\section{EVALUACIÓN Y CLASIFICACIÓN SEGÚN SU PRECISIÓN Y CRITERIO DE SELECCIÓN}

Se realizaron las estimaciones de temperatura por medio de los modelos implementados. Los datos de entrada que se utilizaron corresponden a los obtenidos por medio de un ensayo de elevación de temperatura previo en el que llevando el transformador a pérdidas totales (incluyendo pérdidas sin carga y pérdidas con carga), se determinó la elevación de la temperatura del aceite superior respecto a la temperatura ambiente $\Delta \theta_{\text {or }}$ y la elevación de la temperatura de punto caliente respecto a la temperatura del aceite superior $\Delta \theta_{\mathrm{hr}}$; los datos suministrados por las guías de cargabilidad UNE-IEC 60076-7 de 2010 e IEEE C57.91 de 2011 y los valores propios del ensayo realizado como la temperatura ambiente $\theta_{a}$ durante la prueba.

Es importante acotar que en trabajos similares desarrollados anteriormente, también se han utilizado metodologías experimentales de este tipo para la validación de modelos térmicos, tal es el caso de los transformadores de distribución encapsulados con ventilación natural (Radakovic, 2017). En la Tabla 3 se pueden observar los datos de entrada utilizados para la estimación de temperatura en los modelos. 

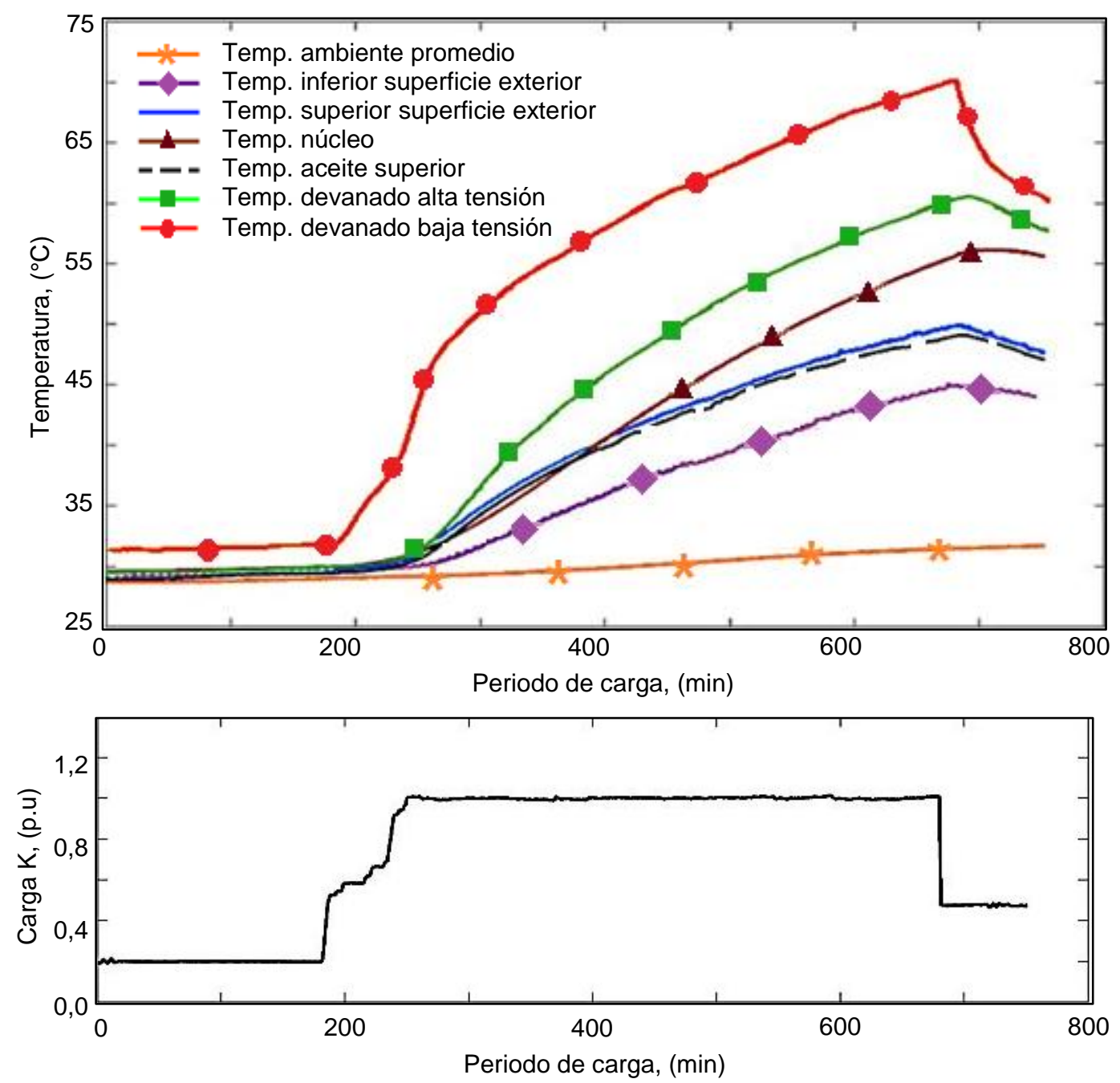

Fig.4: Transformador 37,5 kVA, registro de temperaturas.

Tabla 3: Datos de entrada para los modelos.

\begin{tabular}{|c|c|c|c|}
\hline \multicolumn{2}{|c|}{ Transformador de 15 kVA } & \multicolumn{2}{|c|}{ Transformador de 37,5 kVA } \\
\hline$\theta_{a}$ & $27,9\left({ }^{\circ} \mathrm{C}\right)$ & $\theta_{a}$ & $27,9\left({ }^{\circ} \mathrm{C}\right)$ \\
\hline$\Delta \theta_{\text {or }}$ & $19,9\left({ }^{\circ} \mathrm{C}\right)$ & $\Delta \theta_{\text {or }}$ & $19,9\left({ }^{\circ} \mathrm{C}\right)$ \\
\hline$\Delta \theta_{\mathrm{oi}}$ & $0,12\left({ }^{\circ} \mathrm{C}\right)$ & $\Delta \theta_{\mathrm{oi}}$ & $0,12\left({ }^{\circ} \mathrm{C}\right)$ \\
\hline$\Delta \theta_{\mathrm{hr}}$ & $8,1\left({ }^{\circ} \mathrm{C}\right)$ & $\Delta \theta_{\mathrm{hr}}$ & $8,1\left({ }^{\circ} \mathrm{C}\right)$ \\
\hline$\Delta \theta_{\mathrm{hi}}$ & $4,4\left({ }^{\circ} \mathrm{C}\right)$ & $\Delta \theta_{\mathrm{hi}}$ & $4,4\left({ }^{\circ} \mathrm{C}\right)$ \\
\hline$\tau_{\mathrm{W}}$ & $4(\mathrm{~min})$ & $\tau_{\mathrm{W}}$ & $4(\mathrm{~min})$ \\
\hline$\tau_{\mathrm{o}}$ & $180(\mathrm{~min})$ & $\tau_{\mathrm{o}}$ & $180(\mathrm{~min})$ \\
\hline$\tau_{\mathrm{TO}, \mathrm{R}}$ & $150(\mathrm{~min})$ & $\tau_{\mathrm{TO}, \mathrm{R}}$ & $150(\mathrm{~min})$ \\
\hline$R$ & 1000 & $R$ & 1000 \\
\hline$D$ & 1 & $D$ & 1 \\
\hline$x$ & 0,8 & $x$ & 0,8 \\
\hline$y$ & 1,6 & $y$ & 1,6 \\
\hline$n$ & 0,8 & $n$ & 0,8 \\
\hline$m$ & 0,8 & $m$ & 0,8 \\
\hline$g r$ & $9\left({ }^{\circ} \mathrm{C}\right)$ & $g r$ & $9\left({ }^{\circ} \mathrm{C}\right)$ \\
\hline$H$ & 1,1 & $H$ & 1,1 \\
\hline$k_{11} k_{11}$ & 1 & $k_{11} k_{11}$ & 1 \\
\hline$k_{21}$ & 1 & $k_{21}$ & 1 \\
\hline$k_{22}$ & 2 & $k_{22}$ & 2 \\
\hline$\Delta \theta_{\mathrm{H} / \mathrm{A}, \mathrm{R}}$ & $40\left({ }^{\circ} \mathrm{C}\right)$ & $\Delta \theta_{\mathrm{H} / \mathrm{A}, \mathrm{R}}$ & $40\left({ }^{\circ} \mathrm{C}\right)$ \\
\hline
\end{tabular}

A continuación las Fig. 5 y Fig. 6 se muestran las temperaturas estimadas mediante los tres modelos y la comparación con la temperatura de punto caliente real obtenida, según la carga aplicada en los transformadores de $15 \mathrm{kVA}$ y $30 \mathrm{kVA}$ respectivamente. 

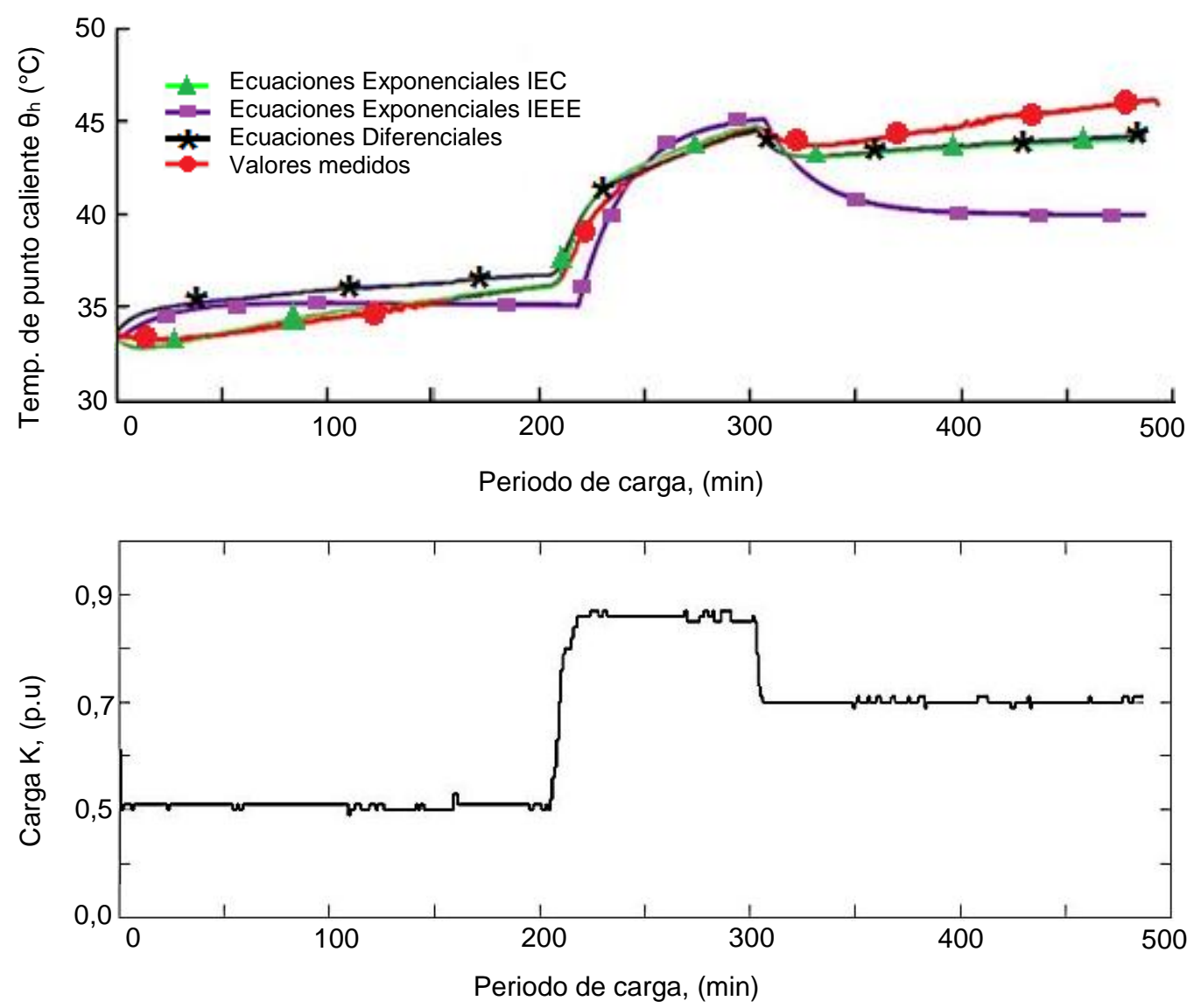

Fig.5: Transformador 15 kVA, comparación temperatura de punto caliente y la carga empleada.
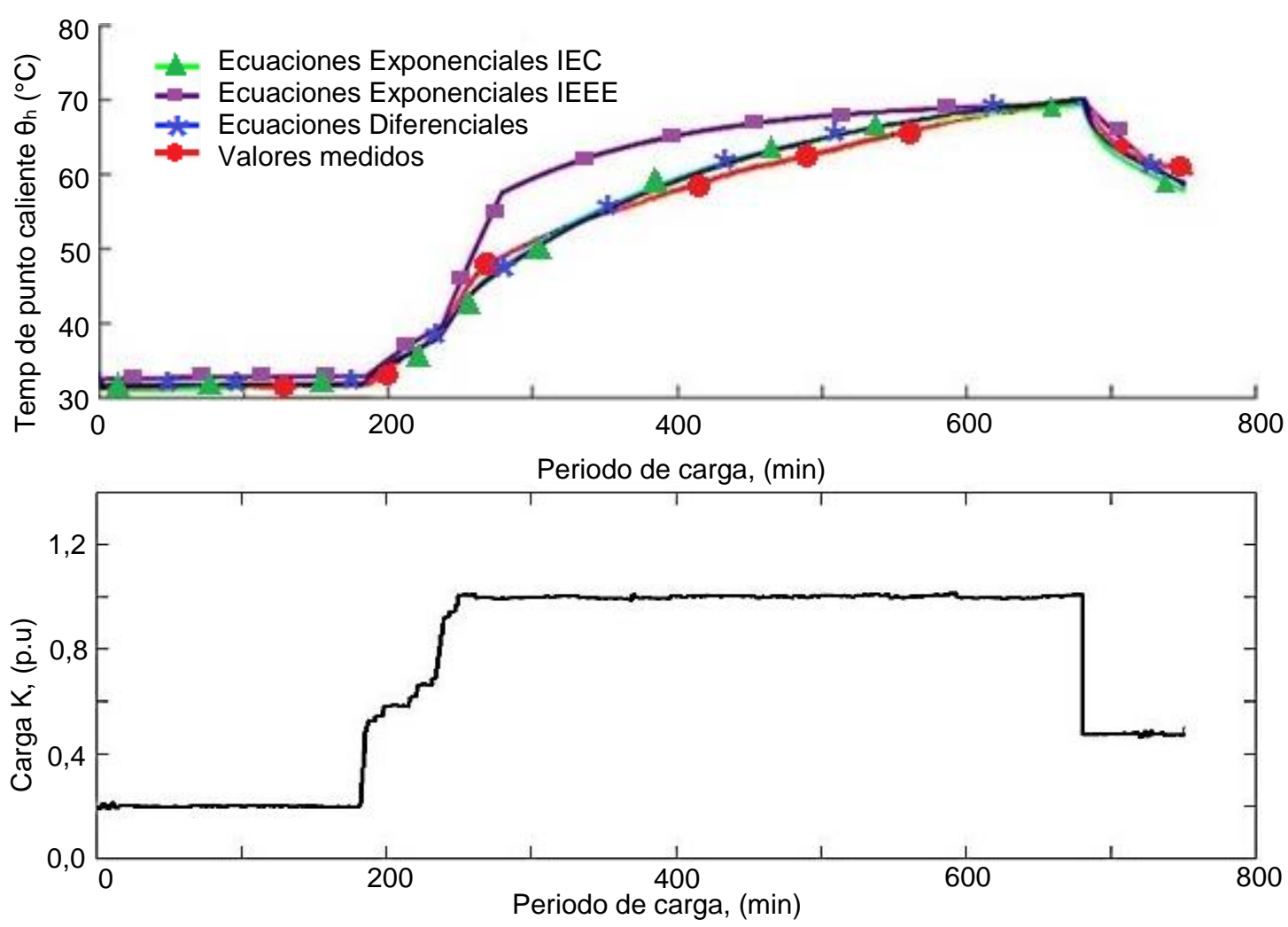

Fig.6: Transformador 37,5 kVA, comparación temperatura de punto caliente.

Como se puede observar en la Fig. 5 y la Fig. 6, el comportamiento arrojado por cada uno de los modelos es muy similar al obtenido mediante las pruebas experimentales, sin embargo, el modelo de ecuaciones diferenciales de IEC es el que presenta un promedio de error menor respecto a los otros modelos estudiados. 
Adicionalmente, el modelo de ecuaciones exponenciales de IEEE presenta los mayores errores máximos los cuales se incrementan con un mayor cambio de carga y temperatura. Dicho comportamiento también ha sido observado en experimentaciones anteriores donde se trata de adaptar el modelamiento térmico de transformadores de potencia a los transformadores de distribución (Susa, 2005), una de las posibles causas atribuibles a este fenómeno es que en los transformadores de distribución la diferencia de las temperaturas del aceite superior e inferior no es tan pronunciada como sucede en los transformadores de potencia, afectando el cálculo del punto caliente implementado en los modelos estandarizados IEEE.

En la Tabla 4 se presentan los errores de las temperaturas estimadas con cada modelo respecto de la temperatura medida en los ensayos. En la Tabla 5 se presentan los errores de las temperaturas estimadas con cada modelo respecto de la temperatura medida en los ensayos.

Tabla 4: Transformador $15 \mathrm{kVA}$, errores máximo y promedio por modelo

\begin{tabular}{|c|c|c|c|c|c|c|}
\hline \multicolumn{3}{|c|}{ Error \% Promedio } & \multicolumn{3}{c|}{ Error \% Máximo } & \multirow{2}{*}{ Carga (p.u.) } \\
\cline { 1 - 6 } Ec. Exp. IEC & Ec. Exp. IEEE & Ec. Dif. IEC & Ec. Exp. IEC & Ec. Exp. IEEE & Ec. Dif. IEC & \\
\hline 0,55 & 2,58 & 3,74 & 2,61 & 7,73 & 5,64 & 0,5 \\
\hline 1,01 & 2,16 & 0,65 & 2,28 & 9,26 & 2,58 & 0,85 \\
\hline 2,61 & 9,08 & 2,44 & 4,41 & 13,37 & 3,98 & 0,7 \\
\hline
\end{tabular}

Tabla 5: Transformador 37,5 kVA, Errores máximo y promedio por modelo.

\begin{tabular}{|c|c|c|c|c|c|c|}
\hline \multicolumn{3}{|c|}{ Error \% Promedio } & \multicolumn{3}{c|}{ Error \% Máximo } & \multirow{2}{*}{ Carga (p.u.) } \\
\cline { 1 - 6 } Ec. Exp. IEC & Ec. Exp. IEEE & Ec. Dif. IEC & Ec. Exp. IEC & Ec. Exp. IEEE & Ec. Dif. IEC & \\
\hline 0,96 & 3,76 & 1,05 & 3,73 & 3,92 & 2,13 & 0,2 \\
\hline 1,81 & 5,24 & 1,31 & 3,22 & 17,78 & 2,86 & 0,6 \\
\hline 1,82 & 6,99 & 1,85 & 3,26 & 17,25 & 4,38 & 1,0 \\
\hline 2,77 & 1,45 & 1,48 & 4,30 & 3,13 & 2,74 & 0,5 \\
\hline
\end{tabular}

Las tablas 4 y 5 denotan un aumento sustancial del error promedio en el paso relacionado con el decremento de temperatura que se estabiliza en 0.7 p.u., destacándose el modelo de ecuaciones exponenciales IEEE con el mayor valor diferencial respecto a los valores reales medidos. En ese sentido se debe tener en cuenta que los transformadores de distribución utilizados durante la experimentación no presentaban un canal de enfriamiento de aceite (radiadores), mientras que el modelo si lo considera.

Este tipo de observaciones también han sido obtenidas en experimentaciones anteriores pero utilizando análogamente el modelo IEC en transformadores de características similares (refrigeración tipo ONAN), en donde dicho modelo ha presentado los máximos valores de error cuando el transformador asume regímenes de carga nominal o sobrecarga (Radakovic, 2003). Por otra parte, también se ha podido establecer que los modelos IEC e IEEE presentan un mejor cálculo del punto caliente para transformadores con enfriamiento tipo ONAF (Nordman, 2003), siendo esta última condición mucho más común en transformadores de potencia.

\section{CONCLUSIONES}

Aunque el modelo de ecuaciones exponenciales de IEC presentó resultados aceptables, requiere conocer la temperatura de punto caliente al menos en un instante durante el periodo de estudio lo cual disminuye la practicidad del mismo. En cuanto al modelo de ecuaciones exponenciales IEEE, este presentó una desviación más significativa en sus datos respecto a la experimentación.

Debido a lo anterior, se determinó que el modelo más viable para aplicar en el cálculo de la temperatura de punto caliente para transformadores es el modelo de ecuaciones diferenciales de IEC, ya requiere un menor número de datos de entrada y a su vez presenta un buen nivel de precisión en sus datos de salida, siempre y cuando se elija un tiempo pequeño para el operador de diferencia D.

\section{AGRADECIMIENTOS}

Los autores desean agradecer a la Universidad del Valle por su apoyo en este trabajo a través del proyecto de investigación 2857: "Validación experimental de un modelo multifísico de difusión de humedadtemperatura para estimación de la dinámica de humedad en el aislamiento celulósico de los transformadores de potencia", y a Colciencias a través del proyecto de investigación 110671550932: "Sistema de monitoreo en línea para la estimación de la vida útil de transformadores de potencia". 


\section{NOTACIÓN}

$\theta_{\mathrm{h}}=$ Temperatura de punto caliente (IEC)

$\theta_{\mathrm{ai}}=$ Temperatura ambiente promedio del intervalo estudiado

$\Delta \theta_{0}=$ Elevación de la temperatura del aceite superior

$\Delta \theta_{\mathrm{h}}=$ Elevación de la temperatura de punto caliente respecto a la del aceite superior

$\Delta \theta_{\text {oi }}=$ Elevaciones de temperatura del aceite superior

$\Delta \theta_{\text {hi }}=$ La temperatura de punto caliente al inicio

$\Delta \theta_{\text {or }}=$ Elevación de la temperatura del aceite superior a pérdidas totales

To $\mathrm{y}_{\mathrm{w}}=$ Constantes de tiempo para la respuesta térmica del aceite y el devanado

$k=$ Factor de carga

$R=$ Relación entre el valor de pérdidas bajo carga y pérdidas sin carga

$\mathrm{k}_{11}, \mathrm{k}_{21}, \mathrm{k}_{22}=$ Constantes empíricas propias del modelo

$\mathrm{x}, \mathrm{y}=$ Constantes empíricas que dependen del tipo de refrigeración del transformador

$\theta_{\mathrm{H}}=$ Temperatura de punto caliente (IEEE)

$\theta_{\mathrm{A}}=$ Temperatura ambiente

$\Delta \theta_{\mathrm{TO}}=$ Elevación de la temperatura del aceite superior sobre la temperatura ambiente

$\Delta \theta_{\mathrm{HI}}=$ Temperatura de punto caliente al inicio

$\Delta \theta_{H}=$ Elevación de la temperatura de punto caliente sobre la del aceite superior

$\Delta \theta_{\text {To }, \text { I }}$ Elevación de la temperatura del aceite superior

$\Delta \theta$ то, $\mathrm{R}=$ Elevación de la temperatura del aceite superior durante pérdidas totales

$K=$ Factor de carga

$n, m=$ Constantes empíricas, su valor depende del tipo de refrigeración del transformador

$D=$ Operador diferencial

\section{REFERENCIAS}

Biçen, Y., Y. Çilliyüz, F. Aras y G. Aydugan, An assessment on aging model of IEEE/IEC standards for natural and mineral oil-immersed transformer, 2011 IEEE International Conference on Dielectric Liquids, 1-4, Trondheim, Noruega, 26-30 de Junio (2011)

Cerón, A.F., D.F. Echeverry, G. Aponte y A.A. Romero, Índice de Salud para Transformadores de Potencia Inmersos en Aceite Mineral con Voltajes entre 69kV y 230kV usando Lógica Difusa, Información Tecnológica, 26(2), 107-116 (2015)

Daghrah, M., Z.D. Wang y otros cuatro autores, Experimental investigation of hot spot factor for assessing hot spot temperature in transformers, 2016 International Conference on Condition Monitoring and Diagnosis (CMD), 948-951, Xi'an, China, 25-28 de Septiembre (2016)

Del Vecchio, R.M., B. Poulin y otros tres autores, Transformer design principles: with applications to core-form power transformers. $2^{\mathrm{a}}$ Ed., 1-21, CRC press, Boca Ratón, USA (2010)

IEEE Std C57.91: IEEE Standards Association, Guide for Loading Mineral-Oil-Immersed Transformers and Step-Voltage Regulators, 1-106, New York, USA (2011)

Navas, D.F., H. Cadavid y D.F. Echeverry, Implementación de un sistema de medición de temperatura empleando fibra óptica para el estudio térmico de transformadores de distribución inmersos en aceite mineral y vegetal, Revista EIA, 10(19), 23-31 (2013)

Nordman, H., N. Rafsback y D. Susa, Temperature Responses to Step Changes in the Load Current of Power Transformers. IEEE Transactions on Power Delivery, 18(4), 1110-1117 (2003)

Radakovic, Z. y K. Feser, A New Method for the Calculation of the Hot-Spot Temperature in Power Transformers With ONAN Cooling, IEEE Transactions on Power Delivery, 18(4), 1284-1292 (2003)

Radakovic, Z., M. Jevtic y B. Das, Dynamic thermal model of kiosk oil immersed transformers based on the thermal buoyancy driven air flow, International Journal of Electrical Power \& Energy Systems, 92(1), 14-24 (2017) 
Santisteban, A., F. Delgado y otros cuatro autores, Numerical analysis of the hot-spot temperature of a power transformer with alternative dielectric liquids, IEEE Transactions on Dielectrics and Electrical Insulation, 24(5), 3226-3235 (2017)

Susa, D. y M. Lehtonen, Dynamic thermal modeling of power transformers: further Development-part II, IEEE transactions on power delivery, 21(4), 1971-1980 (2006)

Susa, D., M. Lehtonen y H. Nordman, Dynamic Thermal Modeling of Distribution Transformers, IEEE transactions on power delivery, 20(3), 1919-1929 (2005)

Taheri, S., A. Vahedi, A. Gholami y H. Taheri, Estimation of Hot Spot Temperature in Distribution Transformer Considering Core Design Using FEM, Power and Energy Conference 2008 (PECon 2008), 1408-1413, Johor Bahru, Malaysia, 1-3 de Diciembre (2008)

Takami, K., M.H. Gholnejad y J. Mahmoudi, Thermal and hot spot evaluations on oil immersed power Transformers by FEMLAB and MATLAB software's, International Conference on Thermal, Mechanical and Multi-Physics Simulation Experiments in Microelectronics and Micro-Systems 2007 (EuroSime 2007), 1-6, Londres, Reino Unido, 16-18 de Abril (2007)

Tang, W.H. y Q.H. Wu, Condition Monitoring and Assessment of Power Transformers Using Computational Intelligence, $1^{\text {a } E d ., ~ 55-70, ~ S p r i n g e r-V e r l a g, ~ L o n d r e s, ~ R e i n o ~ U n i d o ~(2011) ~}$

UNE-IEC 60076-7: Asociación Española de Normalización y Certificación (AENOR), Guía de carga para transformadores de potencia sumergidos en aceite, 1-58, Madrid, España (2010) 
